\title{
Primary Supraclavicular Cystic Lymphangioma in an Adult: A Rare Presentation
}

\author{
Mansoor Tariq Azim1, Syed Mukarram Hussain² and Mohammad Awais Mughal2
}

\begin{abstract}
Cervicothoracic cystic lymphangiomas are found commonly in children less than 2 years of age, but the same are rare in adults. These form as a result of abberant development of embryonic lymph sac which is gradually filled with lymph fluid. ${ }^{1}$ They are commonly found in the head and neck region but can be present anywhere in the body. Preoperative diagnosis is based on careful history of a soft, painless, fluctuant, gradually enlarging mass and imaging. Surgery constitutes the basis of treatment and provides postoperative confirmation of diagnosis via histology. We, here report a case of cervicothoracic cystic lymphangioma in a 24-year male. The rationale of reporting this case is to discuss its presentation, diagnosis and surgical management. Moreover, we also want to highlight its scarcity in adults, the swelling being a diagnostic challenge and role of histology in affirmative diagnosis.
\end{abstract}

Key Words: Cystic hygroma, Cystic lymphangioma, Neck masses, Adult.

\section{INTRODUCTION}

Cystic hygromas are soft tissue tumors which were first described by Redenbacker in 1828 and the name "Cystic Hygroma" was devised by Werner in 1834.1,2 These are now believed to develop from an aberrant proliferation of lymphatic vessels resulting from abnormal development of the lymphatic system. In adults, there is no definitive cause yet ascertained, but trauma and upper respiratory tract infection have been suggested in literature as possible triggers. ${ }^{2}$ In this case, there was no identifiable cause and onset was sudden and gradual. Diagnosis in adults poses a greater challenge than in children and can lead to misdiagnosis. Definitive diagnosis is usually based on postoperative histology. ${ }^{3}$

\section{CASE REPORT}

A 24-year male presented with right sided supraclavicular swelling for the last one year. The swelling gradually increased in size and became more prominent over right supraclavicular region. There was no history of trauma, upper respiratory tract infection, fever, tuberculosis, toothache or pus discharge. There was no associated pain, radiation, neurological or vascular compromise. There was no restriction of neck or shoulder movements. Clinical examination showed a soft, fluctuant, lobulated,

1 Department of General Surgery, Combined Military Hospital (CMH), Kohat, Pakistan

2 Department of General Surgery, Combined Military Hospital (CMH), Rawalpindi, Pakistan

Correspondence: Dr. Mansoor Tariq Azim, Department of General Surgery, Combined Military Hospital (CMH), Kohat, Pakistan

E-mail: mansurtariq@gmail.com

Received: July 19, 2018; Accepted: January 21, 2019 non-tender, non-reducible, trans-illuminant, ill-defined, slightly mobile, semi-circular mass measuring $8 \times 5 \mathrm{~cm}$ over the right supraclavicular region (Figure 1).

Chest X-ray showed normal lung fields. Ultrasonography (USG) evaluation revealed an irregular, multilocular, cystic, anechoic lesion on the right side of neck. Contrast enhanced computed tomography (CECT) scan of neck and thorax showed a large non-enhancing hypodense, multiloculated, thin walled, cystic lesion on the right side of the neck with well-defined and distinct margins in the subcutaneous tissues of the right supraclavicular space. Ultrasound-guided, fine needle aspiration cytology (FNAC) showed lymphocytes and histiocytes with a proteinaceous background. Thus, a preliminary diagnosis of cystic hygroma was made and the patient was scheduled for surgical excision. Under general anesthesia, incision was made directly over the cyst along the Langerhan's lines. Subcutaneous plane was developed between the cyst and skin by using skin hooks and applying traction counter traction. Sharp and blunt dissection was carried out with scissors. Absolute hemostasis was maintained by using harmonic scalpel wherever required; and this also helped avoiding injury to vital structures. Complete

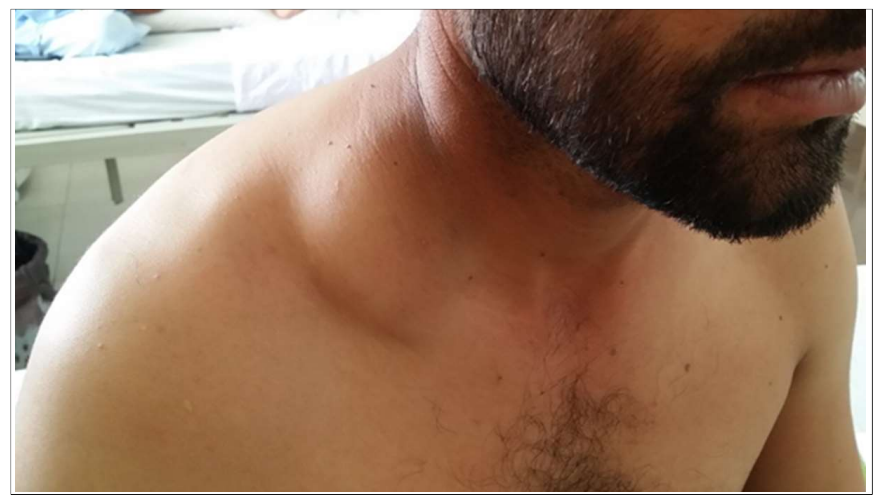

Figure 1: Swelling in the right supraclavicular region. 


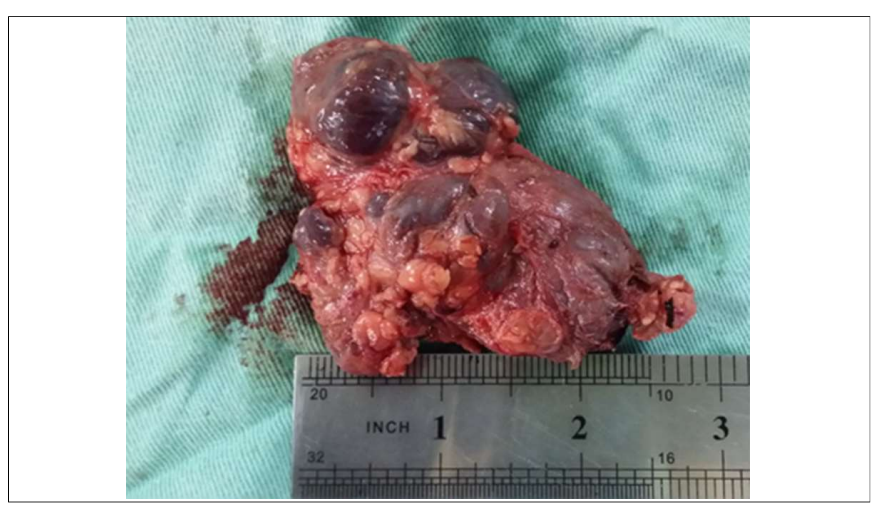

Figure 2: The excised cystic mass

cyst excision was achieved (Figure 2). Histopathological examination of the excised cyst showed fibroadipose tissue containing multiple, variable sized vascular spaces, lined by flattened endothelial cells, consistent with lymphangioma.

\section{DISCUSSION}

Lymphangiomas are benign hamartomatous lesions of lymphatic channels, with marked predilection for head and neck region. They have also been described in a variety of other anatomical locations such as axilla $(20 \%)$, mediastinum (5\%), retroperitoneal space and groin. 4 Hygromas are probably the result of sequestration of lymphatic tissue that has retained its potential for growth. 4 However, the etiology in the adult population is controversial. In literature, it is attributed to delayed proliferation of the congenital or acquired lymphoid vessels as well as following trauma or respiratory infections. There are three histological subtypes of lymphangioms: Capillary lymphangiomas (composed of small lymphatics), cavernous lymphangiomas (composed of larger lymphatics), and cystic lymphangiomas (cystic hygromacomposed of large macroscopic lymphatic spaces with collagen and smooth muscle). Cavernous lymphangiomas are the most common subtype. Most lymphangiomas are asymptomatic. They have no gender predilection and present as painless mass that gradually increases. Typically, the mass is soft, non-tender and ill-defined. Symptoms may develop, when it starts compressing surrounding tissues. There may be obstructive symptoms such as dysphagia, dysphonia, and airway obstruction; however, this is rare in adults. Most cystic hygromas present in-utero or in infancy and, therefore, most of the literature on management considers pediatric cases. ${ }^{4}$ Morsley et al. proposed in his case report that it is essential to evaluate intrathoracic extension of cystic hygroma, which might be seen in $10 \%$ of cases, and this is done by USG/CT/Magnetic Resonant Imaging (MRI). ${ }^{5}$ This patient also underwent CT scan/USG but swelling was restricted to supraclavicular region. Differential diagnosis of the thymic cyst, pericardial cyst, bronchogenic cyst, and cystic teratoma should be kept in mind. ${ }^{4}$

The present case was uncommon, as a cervical cystic hygroma presented de novo in an adult with no history of trauma or upper respiratory tract infection. The treatment of cystic hygroma is primarily surgical. Complete excision of a cystic hygroma has been shown to have an $81 \%$ cure rate. ${ }^{4}$ Non-surgical management includes sclerosants, aspiration, laser surgery, cryotherapy, electrocautery, intralesional steroids, embolisation and radiotherapy. ${ }^{4}$ Sclerotherapy is propagated by some using agents such as OK-432, bleomycin, doxycycline, acetic acid, alcohol, and hypertonic saline. This therapy was not followed for few reasons. First, the success of sclerotherapy has occasionally been measured in terms of sufficient mass reduction without complete resolution. Further, it has been learned that it may not be effective against multiloculated masses, or those of mixed or microcystic anatomy. Moreover, it may also induce a localised immune response which causes a temporary but vivid increase in the size of the mass. ${ }^{6}$

In addition to above, few authors have supported noninvasive management in selected cases to avoid dreary and risky task of surgical resection. Kennedy et al. suggested watchful wait till spontaneous resolution to avoid potential hazards of surgery. ${ }^{7}$ The authors in this case report resorted to complete excision and the recovery was uneventful.

This case contributes to the body of evidence supporting the place of cystic hygroma in the differential diagnosis for cervical masses in an adult. Surgical excision is the treatment of choice for cystic hygromas. Although the tumor is benign, histology must be undertaken for affirmative diagnosis. The goal in the surgical management should be complete excision with good cosmesis.

\section{REFERENCES}

1. Nadour K, Moujahid M. Cervicothoracic cystic lymphangioma: about a case. Pan Afr Med J 2016; 25:189.

2. Bhatia V, Taksande R, Sondankar D. Cystic Hygroma in an Adult Male: A Diagnostic Challenge. Iran J Pathol 2015; 10: 310-3.

3. Gow L, Gulati R, Khan A, Mihaimeed F. Adult-onset cystic hygroma: A case report and review of management. Grand Rounds 2011; 11:5-11.

4. Bahl S, Shah V, Anchlia S, Vyas S. Adult-onset cystic hygroma: A case report of rare entity. Indian J Dent 2016; 7:51-4.

5. Morley SE, Ramesar KC, Macleod DA. Cystic hygroma in an adult: A case report. J R Coll Surg Edinb 1999; 44:57-8.

6. McCaffrey F, Taddeo J. Surgical management of adult-onset cystic hygroma in the axilla. Int J Surg Case Rep 2015; 7:29-3

7. Kennedy TL, Whitaker M, Pellitteri P, Wood WE. Cystic hygrome / lymphangioma: A rational approach to management. Laryngoscope 2001; 111:1929-37. 